\title{
Important cardiac transcription factor genes are accompanied by bidirectional long non-coding RNAs
}

\author{
Yutaro Hori ${ }^{1,2,3}$, Yoko Tanimoto $^{4}$, Satoru Takahashi, ${ }^{4,5}$, Tetsushi Furukawa ${ }^{7}, K^{2}$ Kazuko Koshiba-Takeuchi ${ }^{2,6}$ \\ and Jun K. Takeuchi ${ }^{2,7^{*}}$
}

\begin{abstract}
Background: Heart development is a relatively fragile process in which many transcription factor genes show dosesensitive characteristics such as haploinsufficiency and lower penetrance. Despite efforts to unravel the genetic mechanism for overcoming the fragility under normal conditions, our understanding still remains in its infancy. Recent studies on the regulatory mechanisms governing gene expression in mammals have revealed that long non-coding RNAs (IncRNAs) are important modulators at the transcriptional and translational levels. Based on the hypothesis that IncRNAs also play important roles in mouse heart development, we attempted to comprehensively identify IncRNAs by comparing the embryonic and adult mouse heart and brain.

Results: We have identified spliced IncRNAs that are expressed during development and found that IncRNAs that are expressed in the heart but not in the brain are located close to genes that are important for heart development. Furthermore, we found that many important cardiac transcription factor genes are located in close proximity to IncRNAs. Importantly, many of the IncRNAs are divergently transcribed from the promoter of these genes. Since the IncRNA divergently transcribed from Tbx5 is highly evolutionarily conserved, we focused on and analyzed the transcript. We found that this IncRNA exhibits a different expression pattern than that of Tbx5, and knockdown of this IncRNA leads to embryonic lethality.
\end{abstract}

Conclusion: These results suggest that spliced IncRNAs, particularly bidirectional IncRNAs, are essential regulators of mouse heart development, potentially through the regulation of neighboring transcription factor genes.

Keywords: Heart development, Long non-coding RNA, Haploinsufficiency, Bidirectional promoter

\section{Background}

Morphogenesis is a complex process in which appropriate cell types are differentiated and positioned at the right place and at the proper timing. The surprising reproducibility of developmental processes is underpinned by the robustness of the genetic program [1]. However, in spite of the high robustness under normal genetic conditions, the program can be easily collapsed by genetic abnormalities; for example, some genes require both alleles for proper function (i.e., haploinsufficiency) [2].

\footnotetext{
* Correspondence: juntakeuchi.bip@mri.tmd.ac.jp

${ }^{2}$ Division of Cardiovascular Regeneration, Institute of Molecular and Cellular

Biosciences, the University of Tokyo, Hongo, Bunkyo, Tokyo, Japan

${ }^{7}$ Division of Bio-informational Pharmacology, Medical Research Institute,

Tokyo Medical and Dental University, Tokyo, Japan

Full list of author information is available at the end of the article
}

This type of fragility is frequently observed in mammalian heart development. In the heart, even a slight alteration of the program leads to congenital heart diseases (CHDs) and this fact is associated with the high frequency of CHDs, which is around one in one hundred births [3]. Genetic studies have shown that many of the transcription factor genes involved in the heart development are regulated in a highly spatiotemporal manner [4]. However, how such an intricate control of gene expression is achieved has not been well understood.

Comparative genomics have shown that the complexity of the body plan and the proportion of non-coding regions in the genome are positively correlated [5]. While most of the non-coding regions have previously been considered as "junk", it is now accepted that some

(c) The Author(s). 2018 Open Access This article is distributed under the terms of the Creative Commons Attribution 4.0 International License (http://creativecommons.org/licenses/by/4.0/), which permits unrestricted use, distribution, and reproduction in any medium, provided you give appropriate credit to the original author(s) and the source, provide a link to the Creative Commons license, and indicate if changes were made. The Creative Commons Public Domain Dedication waiver (http://creativecommons.org/publicdomain/zero/1.0/) applies to the data made available in this article, unless otherwise stated. 
of them are necessary for the regulation of genes in a fine and complicated way [6]. Many evo-devo studies support this view, suggesting that the evolution of multicellular organisms was largely driven by the adjustments in transcriptional regulators, such as enhancer elements, rather than by functional evolution of protein-coding genes [7]. Recent advancements in genomics and transcriptomics have demonstrated that nearly half of the mammalian genome is actually transcribed into RNAs [8]. Long non-coding RNA (lncRNA) is an emerging class of RNA that is generally defined as RNAs longer than 200 nucleotides that lack the ability to produce functional proteins. Many of these molecules have been demonstrated to work as transcriptional or translational regulators [9]. Some lncRNAs are known to recruit epigenetic regulators to specific loci in the genome to modulate transcription. For example, a classical lncRNA, Xist, recruits Polycomb repressive complex 2 (PRC2) to the $\mathrm{X}$ chromosome in cis to inactivate one of the two $\mathrm{X}$ chromosomes to achieve dosage compensation [10]. Many lncRNAs studied thus far have been found to bind epigenetic factors and recruit them to defined genomic loci; however, not a small proportion of proposed lncRNA-PRC interactions have been suggested to be non-specific $[11,12]$. Other IncRNAs function as post-transcriptional modulators of gene expression through the formation of duplexes with mRNA to inhibit translation by RNAi (i.e., antisense transcripts) [13], through the inhibition of miRNAs by working as so-called sponges [14] or by controlling splicing [15]. Although much attention has been paid to IncRNAs recently, the low conservation of sequences across species and the difficulty of determining their three-dimensional structures make it difficult to functionally and evolutionarily classify these molecules. Their biochemical characteristics (e.g., strong nonspecific binding to proteins) also make it difficult to dissect their precise molecular functions $[12,16]$. Many lncRNAs show stage- and tissue-specific expression patterns, suggesting their roles in development [17].

Although several lncRNAs that function in mammalian heart development have been reported, the identification and characterization of lncRNAs in the mammalian heart are still insufficient [18-23]. Considering the regulatory nature of IncRNAs, they are thought to be key components in solving the aforementioned problems regarding the developmental fragility in mammalian hearts.

Here, we report that key cardiac transcription factors genes are located in close proximity to genes encoding lncRNAs. Interestingly, there are transcription factor and lncRNA pairs that are bidirectionally transcribed from the same promoter. We have focused on one IncRNA near $T b \times 5$, which we call $T b x 5 u a$, and showed that it is required for heart development. Tbx5ua-knockdown mice showed abnormally thin ventricular walls and were embryonic lethal.

\section{Results}

Identification of IncRNAs that are expressed during mouse heart development

To identify novel lncRNAs that are specifically expressed during heart development in mice, we extracted total RNA from the ventricles of embryonic day (E) 10.5 and E13.5 and 8 weeks-old mice and prepared cDNA libraries, that were subjected to paired-end $2 * 100 \mathrm{bp}$ RNA-seq. The resulting read count was approximately $40 \mathrm{M}$ reads for each sample. The obtained reads were mapped to the mouse genome $(\mathrm{mm} 10)$ with Tophat2 [24], and the mapped reads were assembled using Cufflinks [25] with and without UCSC transcript annotations. Because many of the currently known functional lncRNAs are spliced and because it is difficult to confirm the existence of non-spliced transcripts unless they are expressed at very high levels, we focused on spliced lncRNA candidates in our analysis. We set the lower limit of expression at a fragments per kilobase of exon per million mapped fragments (fpkm) of 1, because above that level, the accuracy of the reconstruction of known transcripts without the transcript reference was sufficiently high (Additional file 1). We also checked if exons of known genes are mistaken as lncRNAs. We found that the direction of a majority of the lncRNAs that are located within 10,000 bp from known genes are in the opposite direction from them (225 vs 86), suggesting that such mis-annotations are rare.

From the assembled transcripts, already known mRNAs or functional RNAs that are not generally classified as lncRNAs (e.g., snoRNA and tRNA) were removed, and we also omitted RNAs that have CDS longer than $1 / 3$ of their total length according to the standard of Ensembl, since they are potentially protein-coding transcripts. As a result, we were able to identify 787 candidates of spliced lncRNAs. To omit lncRNAs that are ubiquitously expressed without tissue specificity, we examined the expression of the obtained candidates in the mouse brain. Because the brain is an organ that diverges from the heart at a very early developmental stage and originates from the ectoderm, whereas the heart originates from the mesoderm, we used the brain as a reference organ. We here just wanted to exclude lncRNAs that are expressed with no tissue specificity and did not intended to find lncRNAs that are exclusively expressed in the heart since many genes are known to function differently according to the context of the tissues. The comparison revealed that 316 of the identified spliced lncRNA candidates were selectively expressed in the heart (Fig. 1a, Additional file 2: Table S1). We checked the expression of these genes in the kidney and the liver and found that only 34 were expressed in both of them, and 213 of them were expressed only in the heart. We found that some lncRNA candidates were expressed in a stage-specific manner, suggesting that they may have roles in heart development or maturation. 


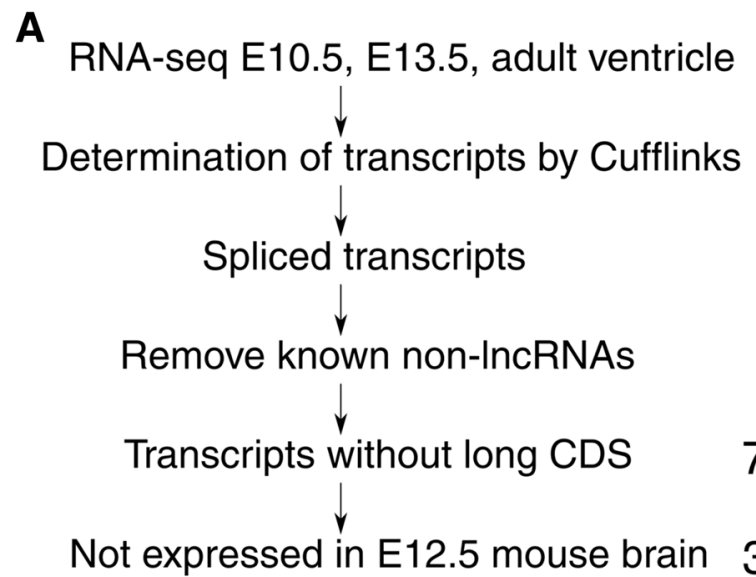

787 candidate loci

B

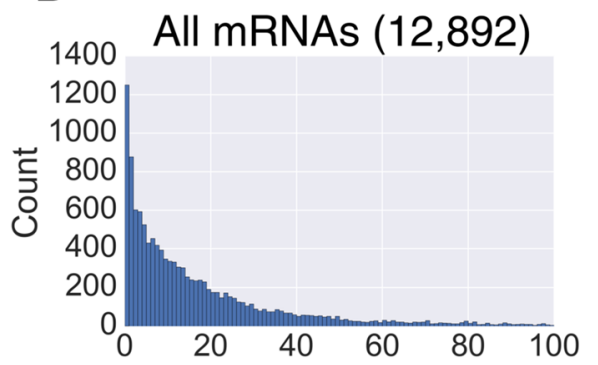

C

All IncRNAs (787)


Fig. 1 The screening procedure of IncRNAs. a The flowchart for the identification of IncRNAs expressed in the mouse heart. $\mathbf{b}$ The histograms of expression levels (fpkm) at E10.5. The number of genes expressed at E10.5 is shown in each parenthesis. The expression levels of IncRNAs are generally lower and IncRNAs with tissue-specificity rarely exceed $10 \mathrm{fpkm}$ (red circle). c The distances from the nearest protein-coding genes were calculated and the distributions were plotted based on RNA-type. We require genes to be expressed in the ventricle at a minimum of one stage. The number of genes in each category is shown in the parenthesis. Heart-selective genes were generally located at greater distances

Many of the cardiac transcription factor genes have neighboring IncRNAs

First, we plotted the distribution of the expression levels of the obtained lncRNAs at E10.5 along with that of mRNAs. Consistent with the previous reports, the expression levels of lncRNAs were much lower than those of mRNAs. Interestingly, almost no heart-selective lncRNAs had fpkm values higher than 10 (Fig. 1b). Since many lncRNAs are known to modulate the transcription of neighboring genes in cis, we tried to identify the 
neighboring genes of the identified lncRNAs. The distribution of the distances from the transcriptional start site (TSS) of lncRNAs to the nearest genes was examined (Fig. 1c). Overall, the distance distribution of all obtained lncRNAs seemed to be similar to that of mRNAs. However, heart-selective lncRNAs were unexpectedly found to be at greater distances to protein-coding genes. The median distances were 12,626, 12,024 and 22,522 for mRNAs, all lncRNAs and heart-selective lncRNAs, respectively $\left(p \approx 3.9 * 10^{-1}\right.$ for all lncRNAs vs. mRNAs; and $p \approx 8.4^{*} 10^{-8}$ for heart-selective lncRNAs vs. mRNAs, Mann-Whitney U test). Next, we examined what types of genes were enriched among the genes closest to lncRNAs. To this end, we conducted a gene enrichment analysis on such protein coding genes using the DAVID bioinformatics tool (http://david.ncifcrf.gov/) [26] and found that transcription factor genes were enriched among genes near lncRNAs in the heart. We also found that the genes associated with heart development were more strongly enriched among the genes near heart-selective lncRNAs when compared to the genes near lncRNAs lacking tissue specificity (Tables 1, 2).

We next tried to identify antisense lncRNAs and lncRNAs from bidirectional promoters. Bidirectional promoters produce two transcripts in a head-to-head divergent manner and attract a lot of attention as important sources of lncRNAs. Preceding studies have revealed that many of them regulate the genes with which they share promoters. We evaluated lncRNAs that had their TSS within $3000 \mathrm{bp}$ from the promoter of protein coding genes as lncRNAs driven by bidirectional promoters. Consistent with the result that the distance between heart-selective lncRNAs and their neighboring genes is generally greater than the distance between all lncRNAs and their neighboring genes, both antisense lncRNA and bidirectional lncRNA were enriched among lncRNAs that are expressed both in the heart and the brain (Fig. 2a) (Additional file 3: Table S2 and Additional file 4: Table S3). Some of the lncRNAs and neighboring genes were judged to be both antisense and bidirectional because of alternative promoter isoforms.

Next, in order to clarify the relationship between mRNAs and their bidirectional lncRNAs, we calculated Pearson correlation coefficients between the $\log 2$-transformed expression levels of the bidirectional promoter pairs over the course of development. The distribution of the correlation coefficients is plotted in Fig. 2b. Many gene pairs clearly show positive or negative correlation, and the positive correlation appears to be dominant (Fig. 2c).

By searching the protein coding genes that are close to lncRNAs, we found many transcription factor genes that have critical functions for heart development (i.e., $T b \times 5$, Tbx20, Nkx2-5, Gata4, Gata6, Sall4, Hand1, Hand2, Wt1, $N r 2 f 1$, Irx3 and Irx5). Notably many of these lncRNAs were bidirectional IncRNAs (i.e., Tbx5, Tbx20, Nkx2-5, Gata6, Sall4, Hand1, Hand2, Wt1, Nr2f1, Irx3 and Irx5). Some of these IncRNAs (e.g., those divergent to Irx5, Gata6 and Wt1) are expressed in the kidney or in the liver, and in such cases divergent genes are also expressed, suggesting that the expression of bidirectional pairs are correlated not only temporally but spatially. We examined the conservation of these IncRNAs near transcription factors by searching the RefSeq database and found that at least some lncRNAs were conserved in the human genome (Tbx5, Nkx2-5, Hand2, Gata6, Wt1 and Nr2f1) (Additional file 5) and that

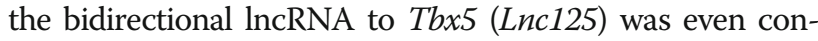
served in chicken, which diverged from mammals 400 million years ago. Here, we judged bidirectional lncRNAs to be conserved solely based on the existence of transcripts at the corresponding loci, since the sequences of lncRNAs are known to evolve rapidly.

Because haploinsufficient transcription factor genes seem to be highly enriched among the genes that are in close proximity to divergent lncRNAs, we determined whether the enrichment was limited to the heart or whether it was more generally true $[27,28]$. Using the mouse RefSeq transcript database (GRCm38.p3) and a paper that comprehensively identified haploinsufficient genes, we tried to determine the proportion of genes with bidirectional lncRNAs among all genes and among haploinsufficient genes [29] (Additional file 6: Table S4). We indeed found that haploinsufficient genes were significantly more enriched among genes with bidirectional lncRNAs $\left(p=3.4 * 10^{-5}\right.$ based on hypergeometric distribution) (Fig. 2d). To exclude the possibility that the tissue specificity of bidirectional lncRNAs and haploinsufficient genes generates pseudo-correlations, we calculated the proportion of housekeeping genes among all genes and among

Table 1 Gene ontology analysis of the genes closest to all IncRNAs that are expressed in the heart

\begin{tabular}{llll}
\hline Term & Count & $\%$ & $p$-value \\
\hline GO:0006355 regulation of transcription, DNA-templated & 86 & 14.42 & $8.51 \mathrm{E}-10$ \\
GO:0006351 transcription, DNA-templated & 72 & 12.07 & $2.09 \mathrm{E}-08$ \\
GO:0045944 positive regulation of transcription from RNA polymerase II promoter & 44 & 7.38 & $5.85 \mathrm{E}-07$ \\
GO:0000122 negative regulation of transcription from RNA polymerase II promoter & 33 & 5.53 & $1.31 \mathrm{E}-05$ \\
GO:0060348 bone development & 8 & 1.34 & $9.50 \mathrm{E}-05$ \\
\hline
\end{tabular}

Transcription factor genes were enriched among the genes close to IncRNAs. GO terms with false discovery rate (FDR) $<0.05$ were sorted by the enrichment $p$-value 
Table 2 Gene ontology analysis of the genes closest to heart-selective IncRNAs

\begin{tabular}{|c|c|c|c|}
\hline Term & Count & $\%$ & $p$-value \\
\hline GO:0045944 positive regulation of transcription from RNA polymerase II promoter & 40 & 9.70 & 2.26E-09 \\
\hline GO:0007507 heart development & 19 & 4.61 & 1.97E-08 \\
\hline GO:0000122 negative regulation of transcription from RNA polymerase II promoter & 31 & 7.52 & $6.80 \mathrm{E}-08$ \\
\hline GO:0051891 positive regulation of cardioblast differentiation & 5 & 6.55 & 1.65E-07 \\
\hline GO:0003151 outflow tract morphogenesis & 8 & 1.94 & $8.14 \mathrm{E}-06$ \\
\hline GO:0060413 atrial septum morphogenesis & 5 & 1.21 & 3.01E-05 \\
\hline GO:0060347 heart trabecula formation & 5 & 1.21 & 4.06E-05 \\
\hline
\end{tabular}

Genes associated with heart development are strongly enriched among genes closest to IncRNAs that are selectively expressed in the heart. Similar terms were omitted

haploinsufficient genes and showed that the proportions were not significantly different (Additional file 7) [30].

Generally, the conservation of lncRNAs across species is very low compared to protein-coding transcripts. However, the $T b x 5$-divergent lncRNA is observed among a wide range of species. $T b \times 5$ is also a dosage-sensitive gene [27]. These findings prompted us to examine the function of the Tbx5-divergent lncRNA.

\section{Analysis of the Tbx5-divergent IncRNA}

$T b \times 5$ is a transcription factor that is known to be essential for the development of the heart and forelimb. Holt-Oram syndrome is a dominant disorder caused by a single-allele mutation of TBX5 and is characterized by hypoplasia of the forelimb, abnormalities in the thumb, and atrial and/or ventricular septal defects [31-33]. Importantly, the phenotypes of Holt-Oram syndrome show a high degree of variance, indicating that the dose of TBX5 is crucial in normal heart development [34].

Hereafter we will call this lncRNA as Tbx5 upstream antisense product (Tbx5ua). Tbx5ua homolog is present in human genome annotation and it is named TBX5-AS1 (Additional file 5). When compared with humans TBX5-AS1 the sequence of Tbx5ua is relatively well conserved at the $5^{\prime}$ region, although it is hard to judge if this conservation is the consequence of functional demand since both the promoter and enhancer elements also exhibit a high degree of conservation (Additional file 8). Tbx5ua is transcribed from one of the promoters of $T b x 5$ in the opposite direction and overlaps with the intron of one of the Tbx5 isoforms (Fig. 3a, RefSeq: XM_006530282.3, isoform 1). RNA-seq data and RefSeq genomic annotation suggest that Tbx5ua is alternatively spliced, producing several isoforms (Fig. 3a, Additional file 9). In Fig. 3a, we labeled isoforms that were identified in our RNA-seq experiment in at least one stage. Reanalysis of previously published intact/nuclear RNA-seq of cardiomyocytes revealed that Tbx5ua is not clearly localized (Fig. 3b) [35]. Previous study reports that quite a few lncRNAs actually exhibit this type of non-localized expression patterns [36].

We first quantified the expression level of the transcript in the heart ventricle, atrium and forelimb during normal development by quantitative RT-PCR (Fig. 3c). We found that the expression level of Tbx5ua was increased in the ventricle as development progressed, which was inconsistent with the expression pattern of $T b x 5$. We also examined the expression level of the $T b \times 5$ isoform that is also transcribed from the bidirectional promoter (Isoform 2, RefSeq: XM_006530280.1). The expression level of that isoform was stable during the entire developmental process, which was also different from the expression pattern of Tbx5ua (Additional file 10). Next, we compared the expression level of the lncRNA in both of the ventricles at E11.5 because it is well-known that the expression level of $T b x 5$ is higher in the left ventricle than in the right ventricle and that the steep gradient is crucial for establishing a proper ventricular septum $[37,38]$. We observed that $T b x 5 u a$ expression was almost the same between the left and right ventricles at E11.5, while we confirmed the differential expression level of $T b x 5$ (Fig. 3d). These results suggest that $T b \times 5 u a$ is not just a byproduct of $T b \times 5$ and is regulated separately as a different product.

\section{Tbx5ua-knockdown (KD) mice were embryonic lethal with severe abnormalities in the heart}

To determine the function of $T b x 5 u a$, we knocked down both alleles of Tbx5ua by inserting three tandem copies of bovine growth hormone polyadenylation site (3xpA) at the second exon to prematurely stop transcription in C57BL/6 J-derived ES cells using the CRISPR/Cas9 system (Fig. 3e) [39]. By tetraploid complementation, we obtained completely ES cell-derived mouse embryos from two ES cell lines and their phenotypes were consistent between lines. The expression level of Tbx5ua in E9.5 KD mice was strongly repressed to approximately $1 / 10$ of that in control embryos, showing successful knockdown (Fig. 4a). Although the expression levels of Tbx5 and Tbx5ua seemed to be anticorrelated in the heart during development (Fig. 3c), KD of Tbx5ua did not result in the increase of $T b x 5$ expression level. We also showed that the expression levels of the different $T b \times 5$ isoforms that are transcribed from all three promoters were not significantly changed (Additional file 11). 
A

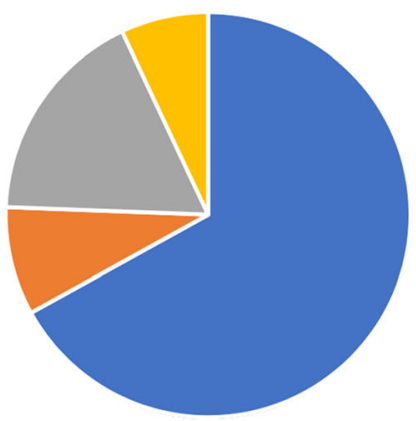

Heart-selective IncRNAs

B

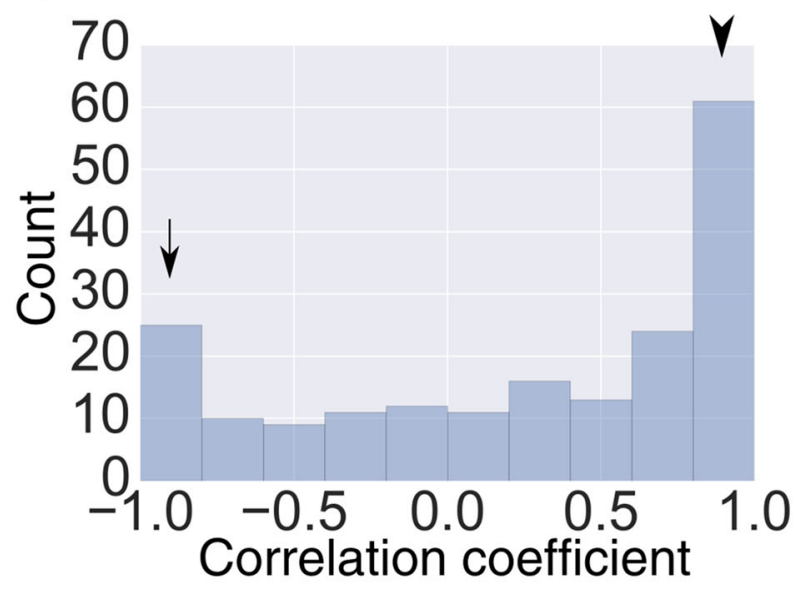

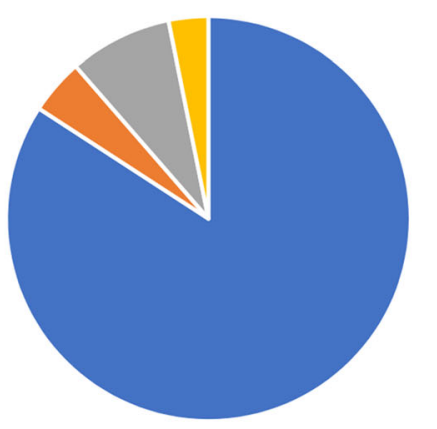

-Neither

Antisense

-Bidirectional

घ Both

D

The proprotion of genes with bidirectional IncRNAs

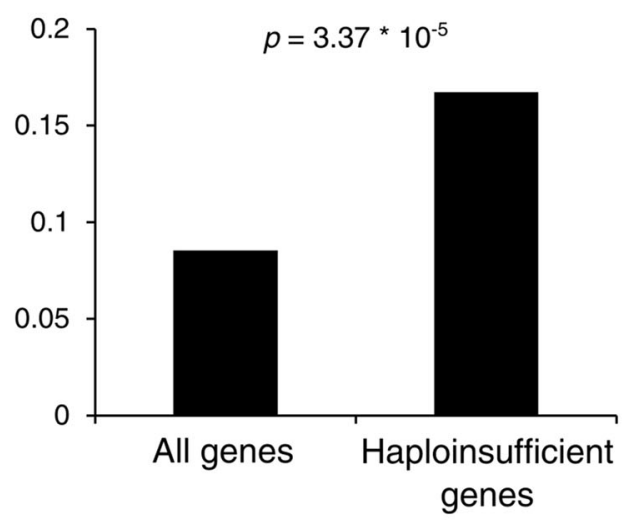

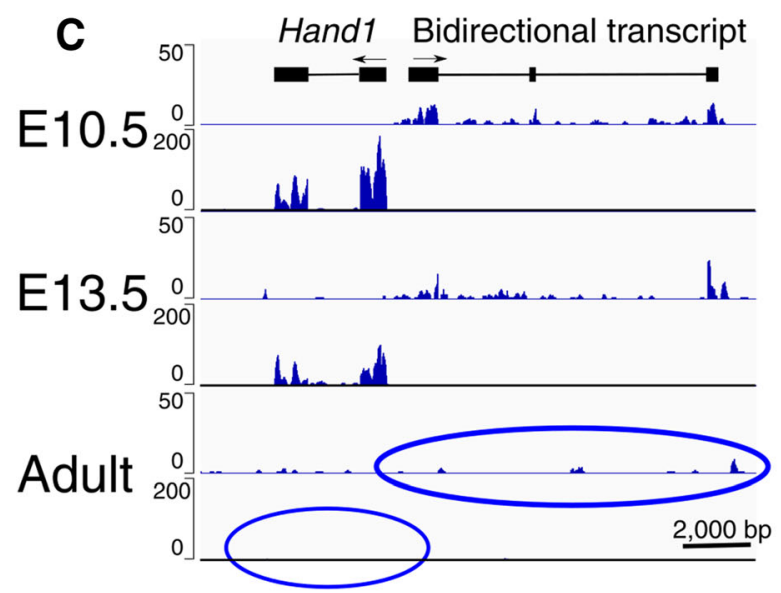

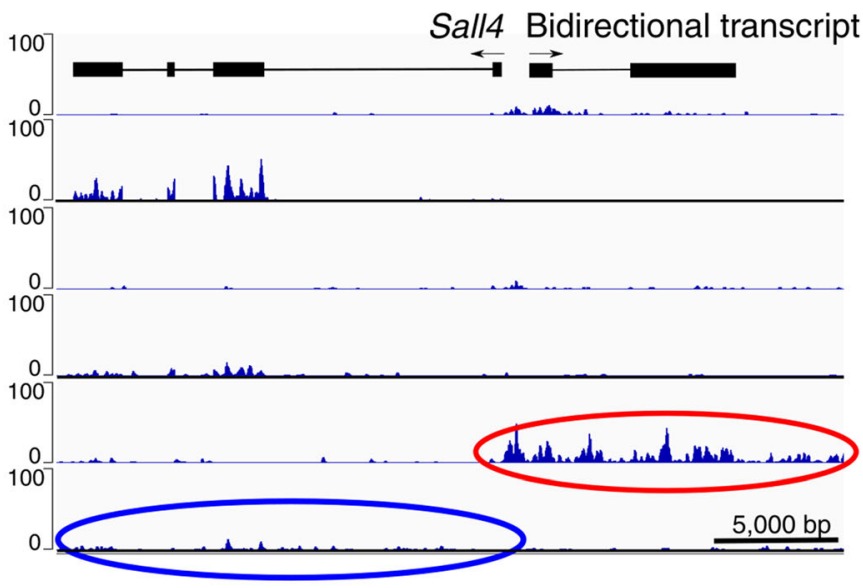

Fig. 2 IncRNAs are enriched near genes that are important for heart development. a Classification of the IncRNA candidates found in the screen. Heart-selective IncRNAs are less likely to be bidirectional or antisense IncRNAs. Some IncRNAs were judged to be both antisense and bidirectional due to alternative promoter isoforms. $\mathbf{b}$ Distribution of the Pearson correlation coefficients between the bidirectional promoter pairs over the course of development. The arrow indicates a negative correlation and the arrowhead indicates a positive correlation. c Hand1 shows a correlated expression pattern with its bidirectional IncRNA (left), while Sall4 exhibits the opposite trend (right). d The proportion of genes that possess bidirectional IncRNAs in the mouse were examined by referring to RefSeq and a paper that identified haploinsufficient genes. Bidirectional IncRNAs were significantly enriched among haploinsufficient genes

Chimeric KD embryos showed right ventricular hypoplasia at E9.5 (Fig. 4b, c, Additional file 12A). Hematoxylin and eosin (HE) staining of the cryosections showed that the ventricular walls of E9.5 KD mice were irregular and lacked trabeculae at some parts in the ventricle (Fig. 4d, Additional files 12B and 13). None of the 


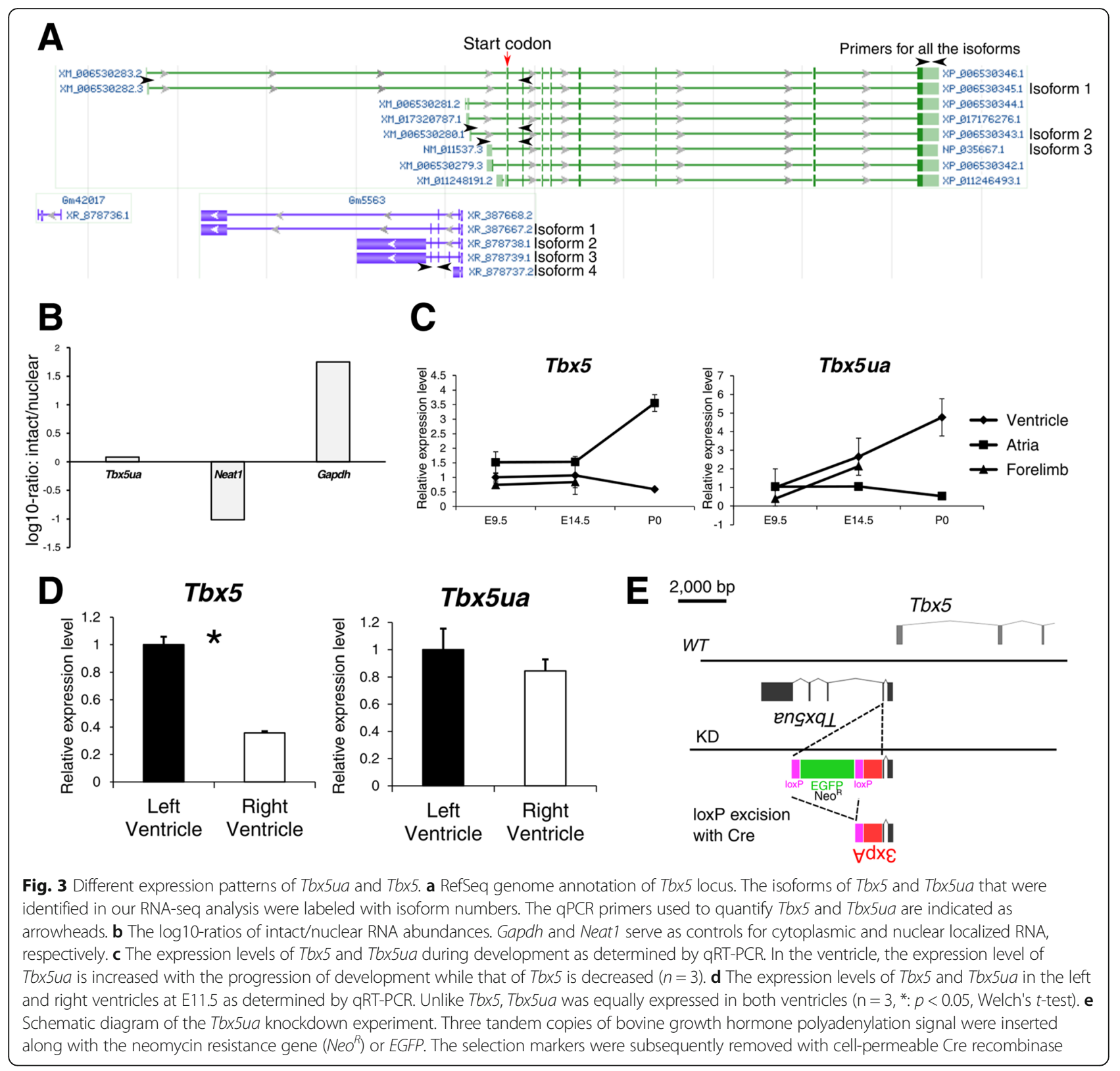

embryos showed a visible abnormality in the forelimbs, which is observed in Tbx5-deficient embryos. By E13.5, all of the KD embryos were dead with a pale body (Fig. 4e). The hearts showed severe ventricular hypoplasia (Fig. 4f), which was probably the cause of the lethality. The forelimbs seemed completely normal even at this stage, which was a significant difference between the phenotype of the Tbx5ua $\mathrm{KD}$ mice and that of the mouse model of Holt-Oram syndrome (i.e., Tbx5 heterozygous knockout) (Fig. 4e). The phenotypes among KD embryos were similar and heart-specific, suggesting that they are attributed to genomic modification. In situ hybridization of $T b \times 5$ revealed normal mRNA expression in the $K D$ ventricle (Additional file 14A). In situ hybridization of $N p p a$, which often shows altered expression pattern in embryos with abnormal morphogenesis, showed an expanded expression around the pre-ventricular septal region of KD embryos (Additional file 14B).

To comprehensively investigate the genes affected by Tbx5ua knockdown, we performed RNA-seq with the RNAs extracted from the ventricles of tetraploid chimeric embryos derived from either KD or WT ES cells. We used three embryos for each group and used the Smart-Seq2 protocol to generate libraries from the small amount of RNA. By gene ontology analysis, we found that the genes 


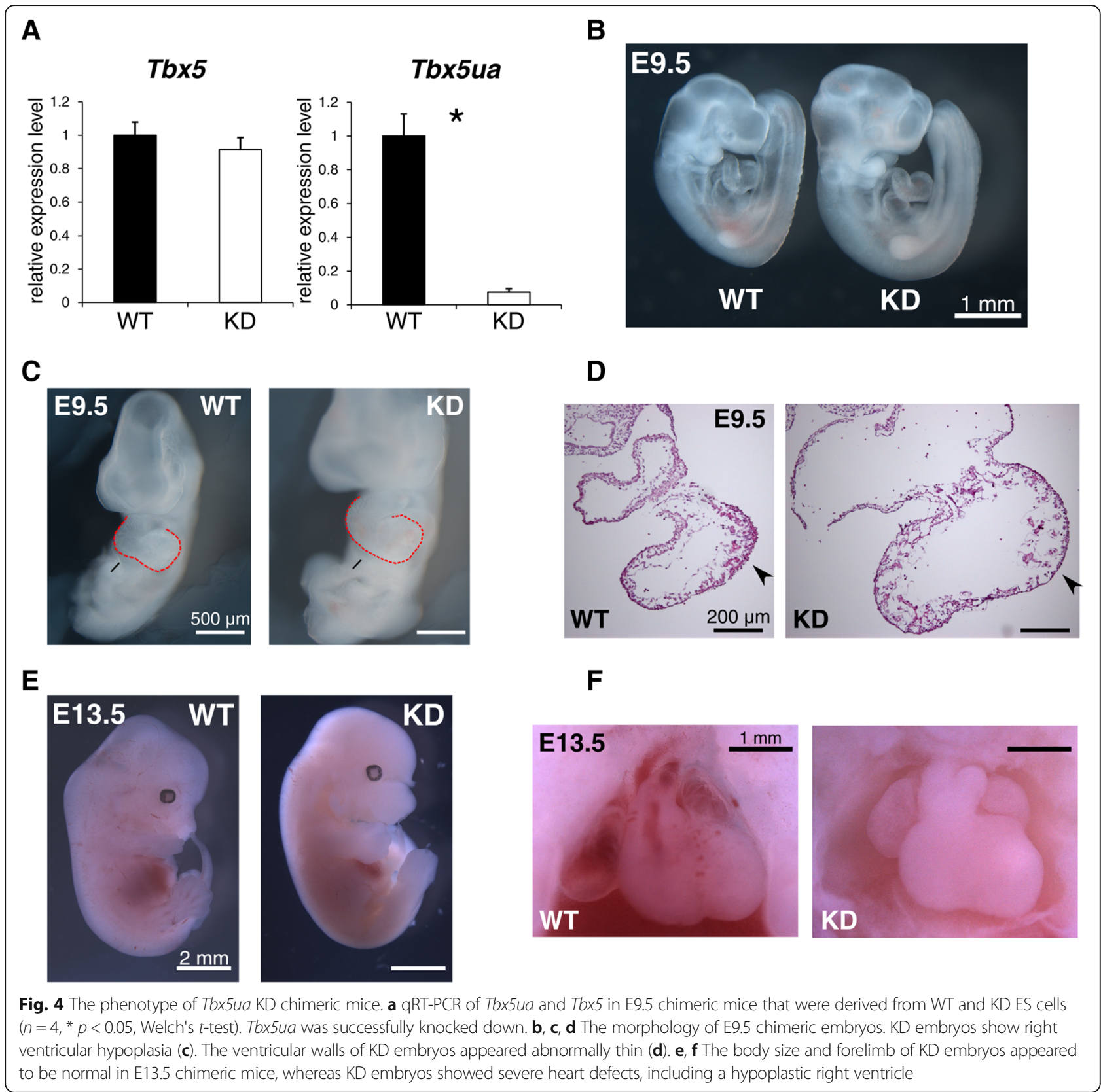

involved in heart development were significantly enriched among the genes that were determined to be significantly changed (False Discovery Rate; FDR $<0.10$, Additional file $15 \mathrm{~A})$. However, none of the structural genes that are important for cardiomyocyte contraction were changed (Additional file 15C), suggesting the possibility that Tbx5ua has a critical role in morphogenesis rather than in cell differentiation. Finally, we conducted principal component analysis (PCA) on the RNA-seq data (Additional file 15D). The two groups were evidently distinguished only by considering the first principal component.

\section{Discussion}

In this study, we found that many cardiac transcription factor genes have neighboring spliced lncRNAs, especially bidirectional ones. The clear correlation of the expression levels of some bidirectional pairs suggests their regulatory roles. A typical example of such lncRNAs is Upperhand, which is divergent to Hand2 [18]. The transcription of Upperhand but not the mature transcripts were shown to be necessary for the transcription of Hand2 by altering the local epigenetic environment. Many lncRNAs are known to regulate local transcription 
through the recruitment of epigenetic-altering protein complexes. Since the expression level of transcription factors is generally low and many of them are haploinsufficient, even a relatively small fluctuation could lead to severe consequences. It is possible that divergent lncRNAs are enriched among dose-sensitive genes to stabilize the expression level of adjacent genes.

An alternative hypothesis is that these transcription factors could be setting up optimal transcriptional environments for lncRNAs to evolve. As some transcription factor genes can cause direct lineage reprogramming, they are thought to define cell types. Thus, the use of these preexisting transcriptional environments is a cost-efficient way to evolve cell type-specific lncRNAs. Some studies have demonstrated that bidirectional transcription is a general phenomenon and that a so-called transcription ripple effect exists [40, 41]. These findings also support our idea by showing that the preexisting transcriptional environment enables precursor transcripts to evolve into defined, functional ones. In summary, active transcription factor genes may have been good sources from which lncRNA genes could evolve due to the cell lineage-specific and active epigenetic environment.

We showed that Tbx5ua is conserved from mammals to birds. Comparison of the sequence of Tbx5ua between mouse and chicken showed less similarity, but it does not mean that the function is not conserved as the previous studies have shown that precise conservation at the sequence level is not necessarily required for the functional conservation of IncRNAs [42, 43]. Tbx5ua was not found in the NCBI genomic annotations of reptiles, amphibians or fish at the corresponding loci. In fact, by conducting the reanalysis on the publicly available RNA-seq data, including RNA-seq of the adult heart of chicken, anole and frog (GSE41338) [44], we could confirm that Tbx5ua is expressed only in chicken among these species at the adult stages (Additional file 16). It is interesting that Tbx5ua is conserved in two-ventricle animals, which possess a complete ventricular septum, but not in animals with non-septated hearts. There is a possibility that the acquisition of Tbx5ua might have contributed to the evolution of a complete ventricular septum.

We showed that Tbx5ua lncRNA is required for proper heart development. Since we knocked down Tbx5ua by prematurely terminating the transcription, the loading of transcription complex at the transcription start site is not inhibited. Thus, if the transcription of Tbx5ua itself is important for altering the local transcriptional environment, our KD scheme is not sufficient to assess the true function of Tbx5ua. Although preliminary, our data suggested that the expression pattern of Tbx 5 protein is altered in the KD mice (Additional file 17). While we do not have any evidences supporting the direct roles of Tbx5ua on Tbx5, the function of Tbx5ua might be atypical for a divergent lncRNA since many of such lncRNAs like Upperhand are shown to alter the transcription of neighboring genes. How the left-sided expression of $T b x 5$ is regulated is an unsolved important issue to understand the molecular mechanism of heart development [45].

\section{Conclusions}

This study revealed that many genes involved in the heart development, particularly transcription factor genes, are associated with spliced lncRNAs that are derived from nearby genomic regions. Furthermore, many of these lncRNAs were divergently transcribed from the promoter of protein-coding genes. We find that bidirectional lncRNAs are enriched among haploinsufficient genes, suggesting that they have functional roles for the regulation of dose-sensitive genes.

\section{Methods \\ RNA-seq}

Total RNAs from embryonic and adult mice were extracted with Sepasol-RNA I Super G (Nacalai \#0937955). The cDNA libraries for paired-end RNA-seq for the screening of lncRNAs were prepared from $1 \mu \mathrm{g}$ of RNAs with Truseq Stranded Total RNA Library Prep Kit (Illumina \#RS-122-2201) according to Illumina's instructions. The cDNA libraries for tetraploid chimeric mice were prepared by Smart-Seq 2 protocol according to the original paper [46] with 12 cycles of preamplification and 9 cycles of enrichment PCR.

\section{qRT-PCR}

Total RNAs were extracted with Sepasol-RNA I Super G (Nacalai \#09379-55). cDNA samples were prepared using RevaTra Ace qPCR RT Master Mix with gDNA remover (Toyobo \#FSQ-301). Real-time PCR was performed with SYBR Premix EX Taq II (Takara \#RR820). The PCR conditions were as follows: $95^{\circ} \mathrm{C}$ for $30 \mathrm{~s}$ followed by 50 cycles of $95^{\circ} \mathrm{C}$ for $5 \mathrm{~s}$ and $60^{\circ} \mathrm{C}$ for $30 \mathrm{~s}$, and a subsequent dissociation curve measurement. We used Gapdh as an internal control. Gene-specific primers are listed in the Additional file 18: Materials and Methods.

\section{Mice}

C57BL/6 J mice were purchased from CLEA Japan. For the first round of chimeric mice generation, we used mice form CLEA Japan. For the second experiment, we used tetraploid embryos from Ark Resource and recipient mice from Sankyo Labo Service. Mice are sacrificed by cervical dislocation. 


\section{Generation of genome edited ES cells}

ES cells were cultured on the MEF feeder in ES culture medium (i.e., Knockout DMEM (Gibco \#10829018), 20\% Knockout Serum Replacement (Gibco \#10828028), 1 * GlutaMAX (Gibco \#10566016), 1 * NEAA (Sigma \#M7145), 1 $\mathrm{mM}$ sodium pyruvate (Gibco \# 11360070), $10^{-4} \mathrm{M}$ 2-Mercaptoethanol, $1000 \mathrm{U} / \mathrm{ml} \mathrm{LiF}$ (Wako \#198-15,781)).

The guide RNA (gRNA) target sequence to induce double strand break is 5'-GTCACTGCCGCTCCAATCC TCGG-3'. We designed the gRNA with Cas-OFFinder (http://www.rgenome.net/cas-offinder/), so that the number of off-target sites was as few as possible. Our gRNA has no potential off-target sites with 0,1 or 2 mismatches and just 4 potential off-targets with 3 mismatches and proper PAM sequence, of them none is exonic. Homology directed repair donors were constructed so that the $N e o^{R}$ or EGFP expressing cassette was flanked by $\sim 1,000$ bp $5^{\prime}$ and $3^{\prime}$ homologous arms cloned from genomic DNA. ES cells were transfected with Cas9 expressing plasmid, gRNA expressing plasmid and the donor plasmid along with non-gRNA expressing negative control. After two days, the ES cells were passaged onto SNL feeder cells and cultured for 8 days with $250 \mu \mathrm{g} / \mathrm{ml}$ G418 and surviving EGFP-positive colonies were manually picked up. After one more cycle of single colony picking up to ensure that the ES cells are clonal, they were subjected to cell permeable Cre treatment [47] to remove the selection cassettes, and then EGFP-negative colonies were picked up to obtain cells without selection cassettes. Finally, the ES cells from each colony were genotyped and karyotyped.

\section{Generation of tetraploid chimeric mice}

Generation of chimeric mice was performed as described previously [39].

\section{Histology}

Immunohistochemistry for Tbx 5 were performed as follows. Antigen retrieval was performed by microwaving the sections in $10 \mathrm{mM}$ citrate acid $\mathrm{pH}$ 6.0. Then they were permeabilized for $10 \mathrm{~min}$ in $0.2 \%$ Triton $\mathrm{X}$ in PBS at RT. Blocking was performed with $10 \%$ Blocking One (Nacalai \#03953-95) in PBST. Tbx5 antibody (Santa Cruz Biotechnology \#sc-17,866) was diluted 1/100 in 5\% Blocking One/PBT and second antibody (Invitrogen \#A-11037) was diluted 1/200.

In situ hybridization was performed as follows. First, cryosections were permeabilized in $0.2 \mathrm{~N} \mathrm{HCl}$ for 15 min. After washing with PBT three times, the sections were re-fixed with freshly made $4 \%$ PFA for $15 \mathrm{~min}$. After washing, the sections were hybridized with DIG labeled probes at $70^{\circ} \mathrm{C}$ for $\mathrm{ON}$. The next day, the sections were washed with $0.2 \times$ SSC three times. After blocking the sections with $10 \%$ sheep serum for $1 \mathrm{~h}, 1 / 1000$ diluted anti-DIG-AP Fab fragment (Roche \#11093274910) were added and incubated for an hour at RT. After washing with TBST, the sections were washed with NTMT and colored with BM purple.

\section{Additional files}

\begin{abstract}
Additional file 1: We counted the exon numbers of reconstructed transcripts and compared them with known exon numbers. The exon numbers were determined based on the maximum of alternative transcripts for each gene. We only took into account genes with their exon number 12 or less since the exon numbers of more than $98.5 \%$ of known IncRNAs expressed in the heart fall under the category. The relation between exon number differenced and fpkm was fitted with an exponential curve. This result demonstrates that $1.0 \mathrm{fpkm}$ is sufficient to infer gene models in our RNA-seq experiment. (PNG $127 \mathrm{~kb}$ )
\end{abstract}

Additional file 2: Table S1. List of spliced IncRNA candidates that were identified in this study. (PDF $204 \mathrm{~kb}$ )

Additional file 3: Table S2. List of antisense IncRNA candidates and their corresponding protein-coding genes. (PDF $17 \mathrm{~kb}$ )

Additional file 4: Table S3. List of bidirectional IncRNA candidates and their corresponding protein-coding genes. (PDF $21 \mathrm{~kb}$ )

Additional file 5: Conserved bidirectional InCRNAs in human USCS genome annotation are shown. We found that many of the mouse IncRNAs divergent to important cardiac transcription factor genes have conserved transcripts at the corresponding loci in human genome. (PNG 733 kb)

Additional file 6: Table S4. List of bidirectional IncRNA candidates that were identified from the analysis of the NCBI RefSeq database (GRCm38.p3). (PDF $148 \mathrm{~kb}$ )

Additional file 7: The proportion of housekeeping genes among all genes and among haploinsufficient genes was calculated and it was not found to be significantly correlated. This result eliminates the possibility that the enrichment of genes with bidirectional IncRNAs among haploinsufficient genes is due to the pseudo-correlation generated through housekeeping-haploinsufficient correlation. (PNG 53 kb)

Additional file 8: The alignment of mouse Tbx5ua (isoform 2) and its human homolog (RefSeq: NR_038440.1) produced by EMBOSS water. The sequence shown in red is highly conserved as determined by EMBOSS Matcher. The sequences are highly conserved at the 5' side. (PDF $158 \mathrm{~kb}$ )

Additional file 9: The sequence of each isoform of Tbx5ua as determined by cufflinks. Isoform numbers correspond to those in Fig. 3a. (PDF 46 kb)

Additional file 10: $q R T-P C R$ analysis of a Tbx5 isoform that is transcribed from the promoter that also produces Tbx5ua (isoform 2). The expression pattern of this isoform over development is also inconsistent with that of Tbx5ua, indicating that they are post-transcriptionally modulated or the directional of transcription is somehow controlled. (PNG $50 \mathrm{~kb}$ )

Additional file 11: $q R T-P C R$ analysis of $K D$ and $W T$ mouse ventricles for all the Tbx5 isoforms detected in our RNA-seq analysis. The expression levels are not significantly changed for all the isoforms. The isoform numbers are indicated in Fig. 3a. (PNG 72 kb)

Additional file 12: Morphological phenotype of Tbx5ua KD embryos derived from another ESC line is shown and is consistent with our first ESC line. (PNG $2047 \mathrm{~kb}$ )

Additional file 13: The thickness of the ventricular wall around the interventricular zone was measured for $\mathrm{WT}$ and $\mathrm{KD}$ embryos and KD embryos tended to have thinner wall. (B) (PNG 44 kb)

Additional file 14: In situ hybridization of Tbx5 and Nppa in WT and KD chimeric mice at E9.5. The expression pattern of Tbx5 at the mRNA level appeared to be not changed. KD embryos showed an ectopic expression of Nppa around the pre-ventricular septal region, which is frequently observed among embryos with abnormal development of ventricular septum. (PNG $6143 \mathrm{~kb}$ )

Additional file 15: RNA-seq analysis of WT and KD chimeric embryos at E9.5 $(n=3)$. (A) Genes related to heart development were enriched 
among genes that were changed significantly. (B) Structural protein genes were not changed, suggesting that the KD did not affect the differentiation of cardiomyocytes in a major way. (C) The scatter plot of log2-transformed expression levels shows that the expression pattern of KD embryos did not change drastically. (D) Principal component analysis on the RNA-seq analysis. WT and KD mice are distinguishable only by the first component. (PNG $767 \mathrm{~kb}$ )

Additional file 16: Reanalysis of RNA-seq data from chicken, anole and zebrafish. Tbx5ua is conserved only in chicken, which possesses a complete ventricular septum, among these species. (PNG $107 \mathrm{~kb}$ )

Additional file 17: $\mathrm{Tb} \times 5 \mathrm{HC}$ of $\mathrm{WT}$ and $\mathrm{KD}$ embryos were quantified. The ventricle was divided into three regions and the staining intensity in each nuclear was measured using ImageJ. Nuclear binary masks were produced from DAPI staining. Note that we only quantified cells that are not in the outermost layer of the ventricle because speckle-like background was observed in the region. Mann-Whitney $\mathrm{U}$ test was performed for each sample with multiple testing correction with Holm method (*: $p<0.05)$. Tbx 5 expression is vanished in the interventricular zone and diminished in the left ventricle in KD embryos. (PNG $1201 \mathrm{~kb}$ )

Additional file 18: Materials and Methods. List of GPCR primers used in this study. (XLSX $11 \mathrm{~kb})$

\section{Abbreviations}

CHD: congenital heart disease; fpkm: fragments per kilobase of exon per million mapped fragments; gRNA: guide RNA; KD: knockdown; IncRNA: long non-coding RNA; Tbx5ua: Tbx5 upstream antisense product; TSS: transcription start site; WT: wildtype

\section{Acknowledgements}

We would like to thank Fumihiro Sugiyama for generously sharing the B6J-ES cells; Akitsu Hotta for kindly sharing the Crispr/Cas9 plasmids; Kikuko Takeuchi and Masao Takeuchi for instructions on karyotyping; Yuri Nakagawa, Yuki Kato and Katsuhiko Shirahige for conducting RNA-seq. We are grateful to prof. Atsushi Miyajima for helpful discussions regarding this work. We also thank the animal centers of the University of Tokyo and the University of Tsukuba.

\section{Funding}

The research was supported by Grants-in-Aid for JSPS Research fellowship $(\mathrm{YH})$, by the Takeda Science Foundation (JKT), by JSPS through the "Funding Program for Next Generation World-Leading Researchers (Next Program)", initiated by the Council for Science and Technology Policy (CSTP; KKT) and by Grants-in-Aid for Scientific Research from the MEXT (Ministry of Education, Culture, Sports, Science and Technology of Japan; JKT). The funding body had no role in the design of the study and collection, analysis, and interpretation of data.

\section{Availability of data and materials}

The datasets supporting the conclusions of this article are available through the NCBI Gene Expression Omnibus (GEO) repository under the accession GSE93324 and GSE93357 (http://www.ncbi.nlm.nih.gov/geo/).

\section{Authors' contributions}

YT and JKT performed the tetraploid complementation assay under the direction of ST and TF. SF and TF also helped the study of tetraploid aggregation. $\mathrm{YH}$ performed all of the other experiments and analyses. $\mathrm{YH}$ wrote the manuscript with the help of KKT and JKT. KKT and JKT supervised the project. All authors read and approved the final version of the manuscript.

\section{Ethics approval and consent to participate}

All experimental procedures and animal care were performed according to the animal ethics committee of the University of Tokyo (2806).

\section{Consent for publication}

Not applicable.

\section{Competing interests}

The authors declare that they have no competing interests.

\section{Publisher's Note}

Springer Nature remains neutral with regard to jurisdictional claims in published maps and institutional affiliations.

\section{Author details}

${ }^{1}$ Laboratory of Cell Growth and Differentiation, Institute of Molecular and Cellular Biosciences, the University of Tokyo, Hongo, Bunkyo, Tokyo, Japan. ${ }^{2}$ Division of Cardiovascular Regeneration, Institute of Molecular and Cellular Biosciences, the University of Tokyo, Hongo, Bunkyo, Tokyo, Japan.

${ }^{3}$ Department of Biological Sciences, Graduate School of Science, the University of Tokyo, Hongo, Bunkyo, Tokyo, Japan. ${ }^{4}$ Laboratory Animal Resource Center, University of Tsukuba, Tsukuba, Ibaraki, Japan. ${ }^{5}$ Department of Anatomy and Embryology, Faculty of Medicine, University of Tsukuba, Tsukuba, Ibaraki, Japan. ${ }^{6}$ Department of Applied Biosciences, Faculty of Life Sciences, Toyo University, Itakura, Gunma, Japan. ${ }^{7}$ Division of Bio-informational Pharmacology, Medical Research Institute, Tokyo Medical and Dental University, Tokyo, Japan.

\section{Received: 3 February 2017 Accepted: 8 November 2018}

\section{Published online: 27 December 2018}

\section{References}

1. Bateson P, Gluckman P. Plasticity and robustness in development and evolution. Int J Epidemiol. 2012;41:219-23.

2. Seidman JG, Seidman C. Transcription factor haploinsufficiency: when half a loaf is not enough. J Clin Invest. 2002;109:451-5.

3. Hoffman JIE, Kaplan S. The incidence of congenital heart disease. J Am Coll Cardiol. 2002;39:1890-900.

4. Srivastava D. Making or breaking the heart: from lineage determination to morphogenesis. Cell. 2006;126:1037-48.

5. Taft RJ, Mattick JS. Increasing biological complexity is positively correlated with the relative genome-wide expansion of non-protein-coding DNA sequences. Genome Biol. 2003;5:P1.

6. Pennisi E. ENCODE Project Writes Eulogy For Junk DNA. Science (80- ). 2012; 337:1159-61.

7. Carroll SB. Evo-devo and an expanding evolutionary synthesis: a genetic theory of morphological evolution. Cell. 2008;134:25-36.

8. Carninci P, Kasukawa T, Katayama S, Gough J, Frith MC, Maeda N, et al. The transcriptional landscape of the mammalian genome. Science. 2005;309: 1559-63.

9. Wang KC, Chang HY. Molecular mechanisms of long noncoding RNAs. Mol Cell. 2011;43:904-14.

10. Brockdorff N. Noncoding RNA and Polycomb recruitment. RNA. 2013;19: 429-42.

11. Khalii AM, Guttman M, Huarte M, Garber M, Raj A, Rivea Morales D, et al. Many human large intergenic noncoding RNAs associate with chromatinmodifying complexes and affect gene expression. Proc Natl Acad Sci U S A. 2009:106:11667-72.

12. Davidovich C, Wang X, Cifuentes-Rojas C, Goodrich KJ, Gooding AR, Lee JT, et al. Toward a consensus on the binding specificity and promiscuity of PRC2 for RNA. Mol Cell. 2015;57:552-9.

13. Faghihi M, Wahlestedt C. Regulatory roles of natural antisense transcripts. Nat Rev Mol Cell Biol. 2009;10:637-43.

14. Ebert MS, Sharp PA. Emerging roles for natural microRNA sponges. Curr Biol. 2010;20:R858-61.

15. Bardou F, Ariel F, Simpson CG, Romero-Barrios N, Laporte P, Balzergue S, et al. Long Noncoding RNA Modulates Alternative Splicing Regulators in Arabidopsis. Dev Cell. 2014;30:166-76.

16. Novikova IV, Hennelly SP, Sanbonmatsu KY. Tackling structures of long noncoding RNAs. Int J Mol Sci. 2013;14:23672-84.

17. Gloss BS, Dinger ME. The specificity of long noncoding RNA expression. Biochim Biophys Acta - Gene Regul Mech. 1859;2015:16-22.

18. Anderson KM, Anderson DM, McAnally JR, Shelton JM, Bassel-Duby R, Olson EN. Transcription of the non-coding RNA upperhand controls Hand2 expression and heart development. Nature. 2016;539:433-6.

19. Klattenhoff CA, Scheuermann JC, Surface LE, Bradley RK, Fields PA, Steinhauser ML, et al. Braveheart, a Long Noncoding RNA Required for Cardiovascular Lineage Commitment. Cell. 2013;152:570-83.

20. Grote P, Wittler L, Hendrix D, Koch F, Währisch S, Beisaw A, et al. The tissuespecific IncRNA Fendrr is an essential regulator of heart and Body Wall development in the mouse. Dev Cell. 2013;24:206-14. 
21. Matkovich SJ, Edwards JR, Grossenheider TC, de Guzman SC, Dorn GW. Epigenetic coordination of embryonic heart transcription by dynamically regulated long noncoding RNAs. Proc Natl Acad Sci U S A. 2014;111: 12264-9.

22. Zangrando J, Zhang L, Vausort M, Maskali F, Marie PY, Wagner DR, et al. Identification of candidate long non-coding RNAs in response to myocardial infarction. BMC Genomics. 2014;15:460.

23. Yang KC, Yamada KA, Patel AY, Topkara VK, George I, Cheema FH, et al. Deep RNA sequencing reveals dynamic regulation of myocardial noncoding RNAs in failing heart and remodeling with mechanical circulatory support. Circulation. 2014;129:1009-21.

24. Kim D, Pertea G, Trapnell C, Pimentel H, Kelley R, Salzberg SL. TopHat2: accurate alignment of transcriptomes in the presence of insertions, deletions and gene fusions. Genome Biol. 2013;14:R36.

25. Trapnell C, Williams BA, Pertea G, Mortazavi A, Kwan G, van Baren MJ, et al. Transcript assembly and quantification by RNA-Seq reveals unannotated transcripts and isoform switching during cell differentiation. Nat Biotechnol. 2010;28:511-5

26. Huang DW, Lempicki RA, Sherman BT. Systematic and integrative analysis of large gene lists using DAVID bioinformatics resources. Nat Protoc. 2009:4:44-57.

27. Moskowitz IPG, Pizard A, Patel W, Bruneau BG, Kim JB, Kupershmidt S, et al. The T-box transcription factor Tbx 5 is required for the patterning and maturation of the murine cardiac conduction system. Development. 2004; 131:4107-16.

28. Jay PY, Rozhitskaya O, Tarnavski O, Sherwood MC, Dorfman AL, Lu Y, et al. Haploinsufficiency of the cardiac transcription factor Nkx2-5 variably affects the expression of putative target genes. FASEB J. 2005;19:1495-7.

29. Dang VT, Kassahn KS, Marcos AE, Ragan MA. Identification of human haploinsufficient genes and their genomic proximity to segmental duplications. Eur J Hum Genet. 2008;16111:1350-7.

30. Eisenberg E, Levanon EY. Human housekeeping genes, revisited. Trends Genet. 2013;29:569-74

31. Holt M, Oram S. Familial heart disease with skeletal malformations. Br Heart J. 1960;22:236-42.

32. Bruneau BG, Nemer G, Schmitt JP, Charron F, Robitaille L, Caron S, et al. A murine model of Holt-Oram syndrome defines roles of the T-box transcription factor Tbx5 in cardiogenesis and disease. Cell. 2001;106: 709-21.

33. Li QY, Newbury-Ecob RA, Terrett JA, Wilson DI, Curtis AR, Yi CH, et al. HoltOram syndrome is caused by mutations in TBX5, a member of the Brachyury (T) gene family. Nat Genet. 1997;15:21-9.

34. Mori AD, Bruneau BG. TBX5 mutations and congenital heart disease: HoltOram syndrome revealed. Curr Opin Cardiol. 2004;19:211-5.

35. Preissl S, Schwaderer M, Raulf A, Hesse M, Grüning BA, Köbele C, et al. Deciphering the epigenetic code of cardiac myocyte transcription. Circ Res. 2015;117:413-23.

36. Cabili MN, Dunagin MC, McClanahan PD, Biaesch A, Padovan-Merhar O, Regev A, et al. Localization and abundance analysis of human IncRNAs at single-cell and single-molecule resolution. Genome Biol. 2015;16:20.

37. Takeuchi JK, Ohgi M, Koshiba-Takeuchi K, Shiratori H, Sakaki I, Ogura K, et al. Tbx5 specifies the left/right ventricles and ventricular septum position during cardiogenesis. Development. 2003;130:5953-64.

38. Koshiba-Takeuchi K, Mori AD, Kaynak BL, Cebra-Thomas J, Sukonnik T, Georges RO, et al. Reptilian heart development and the molecular basis of cardiac chamber evolution. Nature. 2009;461:95-8.

39. Tanimoto $Y$, lijima $S$, Hasegawa $Y$, Suzuki $Y$, Daitoku Y, Mizuno S, et al. Embryonic stem cells derived from C57BL/6J and C57BL/6N mice. Comp Med. 2008;58:347-52.

40. Ebisuya M, Yamamoto T, Nakajima M, Nishida E. Ripples from neighbouring transcription. Nat Cell Biol. 2008;10:1106-13.

41. Almada AE, Wu X, Kriz AJ, Burge CB, Sharp PA. Promoter directionality is controlled by U1 snRNP and polyadenylation signals. Nature. 2013;499:360-3.

42. Tripathi V, Shen Z, Chakraborty A, Giri S, Freier SM, Wu X, et al. Long noncoding RNA MALAT1 controls cell cycle progression by regulating the expression of oncogenic transcription factor B-MYB. PLoS Genet. 2013;9: e1003368

43. Okamoto I, Patrat C, Thépot D, Peynot N, Fauque P, Daniel N, et al. Eutherian mammals use diverse strategies to initiate $X$-chromosome inactivation during development. Nature. 2011:472:370-4.
44. Rabbow E, Rettberg P, Barczyk S, Bohmeier M, Panitz C, Horneck G, et al. The Evolutionary Landscape of Alternative Splicing in Vertebrate. Science (80- ). 2012;12:374-87

45. Smemo S, Campos LC, Moskowitz IP, Krieger JE, Pereira AC, Nobrega MA. Regulatory variation in a TBX5 enhancer leads to isolated congenital heart disease. Hum Mol Genet. 2012;21:3255-63.

46. Picelli S, Björklund ÅK, Faridani OR, Sagasser S, Winberg G, Sandberg R. Smart-seq2 for sensitive full-length transcriptome profiling in single cells. Nat Methods. 2013;10:1096-8.

47. Münst B, Patsch C, Edenhofer F. Engineering cell-permeable protein. J Vis Exp. 2009;34:e1627.

\section{Ready to submit your research? Choose BMC and benefit from:}

- fast, convenient online submission

- thorough peer review by experienced researchers in your field

- rapid publication on acceptance

- support for research data, including large and complex data types

- gold Open Access which fosters wider collaboration and increased citations

- maximum visibility for your research: over $100 \mathrm{M}$ website views per year

At BMC, research is always in progress.

Learn more biomedcentral.com/submissions 